\title{
Ampla abordagem sobre a sífilis congênita: revisão narrativa
}

\author{
Broad approach to congenital syphilis: narrative review \\ Enfoque amplio de la sífilis congénita: revisión narrativa
}

Amanda Brandão Lopes ${ }^{1 *}$, Isabella Alvim Werner ${ }^{2}$, Roberta Médice Fonseca ${ }^{2}$, Brunna Aquino de Souza Pinto ${ }^{2}$, Isa Depe ${ }^{2}$, Henrique Santos Fernandes ${ }^{3}$, Marcelo de Souza Mangini ${ }^{4}$, Thiago de Moraes Vilela ${ }^{5}$, Patrícia Pimenta Nunes ${ }^{1}$, Mellissa Aleixo Machado².

\section{RESUMO}

Objetivo: Fornecer através de uma revisão narrativa uma ampla abordagem sobre a Sífilis Congênita (SC). Revisão bibliográfica: A sífilis é uma doença bacteriana causada pelo Treponema Pallidum que pode ser transmitida de forma sexual ou vertical. No caso da SC, o bebê contrai a doença através da placenta ainda no útero da mãe contaminada, em qualquer estágio da gestação. O diagnóstico é realizado com base na história materna, por isso, um pré-natal de qualidade é determinante no controle da doença. O recém-nascido com SC é, na maioria das vezes, assintomático ao nascimento. A doença é capaz de afetar os diversos sistemas orgânicos e as manifestações clínicas são divididas em precoces e tardias. O tratamento é realizado com penicilina benzatina, procaína ou cristalina a depender do estágio da doença. Considerações finais: A sífilis congênita pode ser facilmente prevenida por meio do pré-natal adequado, onde serão realizados o diagnóstico e tratamento da gestante, evitando dessa forma a transmissão para o feto. Uma vez constatada a SC, o bebê deve ser adequadamente tratado e receber acompanhamento multiprofissional, com o objetivo de evitar ao máximo as sequelas da doença.

Palavras-chave: Sífilis congênita, Infecções congênitas, Sífilis.

\section{ABSTRACT}

Objective: Provide, through a narrative review, a broad approach to Congenital Syphilis (CS). Bibliographic review: Syphilis is a bacterial disease caused by Treponema Pallidum that can be transmitted sexually or vertically. In the case of CS, the baby contracts the disease through the placenta while still in the infected mother's uterus, at any stage of pregnancy. Diagnosis is made based on maternal history, therefore, quality prenatal care is crucial in controlling the disease. The newborn with CS is, in most cases, asymptomatic at birth. The disease is capable of affecting different organic systems and clinical manifestations are divided into early and late. Treatment is carried out with benzathine penicillin, procaine or crystalline depending on the stage of the disease. Final considerations: Congenital syphilis can be easily prevented through adequate prenatal care, where the diagnosis and treatment of the pregnant woman will be carried out, thus avoiding transmission to the fetus. Once CS is found, the baby must be properly treated and receive multidisciplinary follow-up, in order to avoid as much as possible the sequelae of the disease.

Key words: Congenital syphilis, Congenital infections, Syphilis.

\section{RESUMEN}

Objetivo: Proporcionar, a través de una revisión narrativa, un enfoque amplio de la sífilis congénita (SC). Revisión bibliográfica: La sífilis es una enfermedad bacteriana causada por Treponema pallidum que puede

\footnotetext{
${ }^{1}$ Faculdade de Minas de Belo Horizonte (FAMINAS-BH), Belo Horizonte - MG.

*E-mail: amandabrandaolopes@gmail.com

${ }^{2}$ Faculdade de Ciências Médicas e da Saúde de Juiz de Fora (SUPREMA), Juiz de Fora - MG.

${ }^{3}$ Centro Universitário UNIFACIG, Manhuaçu - MG.

${ }^{4}$ Faculdade da Saúde e Ecologia Humana (FASEH), Vespasiano - MG.

${ }^{5}$ Universidade de Itaúna (UIT), Itaúna - MG.
} 
transmitirse de forma sexual o vertical. En el caso del CS, el bebé contrae la enfermedad a través de la placenta mientras aún está en el útero de la madre infectada, en cualquier etapa del embarazo. El diagnóstico se basa en la historia materna, por lo que la atención prenatal de calidad es fundamental para controlar la enfermedad. El recién nacido con SC es, en la mayoría de los casos, asintomático al nacer. La enfermedad es capaz de afectar diferentes sistemas orgánicos y las manifestaciones clínicas se dividen en precoces y tardías. El tratamiento se realiza con penicilina benzatínica, procaína o cristalina según el estadio de la enfermedad. Consideraciones finales: La sífilis congénita se puede prevenir fácilmente mediante una adecuada atención prenatal, donde se realizará el diagnóstico y tratamiento de la gestante, evitando así la transmisión al feto. Una vez detectado el SC, el bebé debe ser tratado adecuadamente y recibir un seguimiento multidisciplinario, con el fin de evitar en la medida de lo posible las secuelas de la enfermedad.

Palabras clave: Sífilis congénita, Infecciones congénitas, Sífilis.

\section{INTRODUÇÃO}

As Doenças Sexualmente Transmissíveis (DSTs) ainda são consideradas um problema de saúde pública considerável, sendo mais prevalente nos países em desenvolvimento. São responsáveis, principalmente entre mulheres e crianças, por alguns problemas de âmbitos sociais, econômicos e sanitários de grande proporção. A sífilis ainda é encontrada em um número significativo de gestantes brasileiras (MAGALHÃES DMS, et al., 2013).

A sífilis é caracterizada como uma doença de evolução crônica, do tipo infecciosa e sistêmica causada por uma bactéria conhecida como Treponema Pallidum. Ela é do tipo gram-negativa do grupo das espiroquetas e tem o homem como único hospedeiro, reservatório e transmissor. Sua transmissão pode ocorrer de forma vertical ou sexual. Sendo assim, um pré-natal de qualidade é fundamental para seu controle e prevenção de sua transmissão vertical (MADRID L, et al., 2016).

A probabilidade de transmissão vertical do $T$. pallidum durante a gestação pode ser definida por dois fatores: o tempo de exposição do feto no útero e o estágio da sífilis na gestante. Em mulheres não tratadas, a taxa de infecção da transmissão vertical pode variar nas fases primária e secundária da doença entre 70 a $100 \%$. Já nas fases tardias de infecção, essa taxa pode reduzir para cerca de 30\% (JAAN A e RAJNIK M, 2021).

A sífilis congênita ocorre por transmissão do tipo vertical da espiroqueta, que passa da mãe para o feto através da placenta. Além disso, se houver lesões genitais na mãe, há possibilidade de transmissão direta do T. pallidum através do canal do parto. Já no aleitamento materno, só é possível a transmissão se houver lesão mamária por sífilis (HUSSAIN AS e VAIDYA R, 2021).

As consequências da sífilis para a gestação podem variar desde um parto prematuro assintomático à uma gama de sinais e sintomas clínicos e chegar até o natimorto. Algumas pesquisas estimam que ela é um fator de complicação em aproximadamente 1 milhão de gestações por ano em todo o mundo, sendo grande contribuinte para a morte infantil e sendo responsável, por ano, por cerca de 305.000 mortes perinatais mundialmente (MADRID L, et al., 2016).

Tanto nas gestantes quanto nas não gestantes, as alterações fisiopatogênicas que podem ser observadas são as mesmas, podendo ser também assintomáticas. Como se trata de uma doença de tratamento fácil, a grande maioria dos casos é observada em gestantes que não possuíram cuidados pré-natais adequados ou receberam tratamento inadequado $A$ sífilis congênita pode ser classificada como tardia ou precoce, sendo essa última quando diagnosticada até dois anos de vida e tardia (HUSSAIN AS e VAIDYA R, 2021).

A revisão em questão teve por objetivo fornecer uma ampla abordagem sobre a sífilis congênita com enfoque principal no diagnóstico, manifestações clínicas e tratamento, a fim de promover conhecimento para os profissionais da saúde e para a população em geral para a prevenção desta doença e manejo das crianças acometidas. 


\section{REVISÃO BIBLIOGRÁFICA}

\section{Etiologia da sífilis}

Em 1905, Fritz Schaudinn e Erich Hoffmann isolaram pela primeira vez o microorganismo causador da sífilis. Conhecida por Treponema pallidum, trata-se de uma espiroqueta móvel, helicoidal, medindo cerca de 6 a 15 micrômetros de comprimento e 0,1 a 0,2 micrômetros de largura. Na Europa, mais especificamente na atual Itália, foi o local onde foi registrado o primeiro surto de sífilis, no ano de 1949. Até o momento, essa bactéria ainda não foi cultivada in vitro, sendo o ser humano o seu único hospedeiro conhecido (GALVIS AE e ARRIET A, 2020).

\section{Epidemiologia da sífilis congênita}

É estimado que cerca de um milhão de gestações por ano em todo o mundo seja complicada por decorrência da sífilis, segundo os levantamentos da Organização Mundial de Saúde (OMS), o que acaba colocando mais de 200 mil crianças em risco de morte prematura e levando a mais de 300 mil mortes fetais e neonatais anualmente. Apesar dos esforços mundiais para o combate da sífilis congênita, os dados disponíveis revelam que a afecção continua sendo um problema relevante de saúde pública, principalmente nos países em desenvolvimento, os quais concentram cerca de $90 \%$ dos casos (MINISTÉRIO DA SAÚDE, 2014).

No Brasil, um aumento constante no número de casos de sífilis em gestantes foi observado nos últimos, tanto de sífilis congênita quanto de sífilis adquirida. Esse aumento pode ser atribuído ao aumento do número de diagnósticos precoces que estão sendo estabelecidos, quanto à diminuição do uso de preservativos, assim como ao desabastecimento mundial de Penicilina (MINISTÉRIO DA SAÚDE, 2019).

\section{Transmissão materno-fetal da sífilis}

A bactéria Treponema Pallidum é o agente causador da sífilis. Ela é capaz de chegar até o feto atravessando a placenta, o que é chamada de transmissão vertical. Essa forma de transmissão pode ocorrer em qualquer estágio da doença em que a mãe se encontra e durante qualquer período da gestação, sendo mais comum no último trimestre. Algumas das consequências do acometimento fetal são: prematuridade, natimorto, aborto, morte neonatal e diversas manifestações clínicas que podem surgir de maneira precoce ou tardia. Além disso, alguns recém-nascidos são infectados durante o parto, na passagem pelo canal vaginal e isso ocorre por contato com alguma lesão genital materna. Quanto ao processo de amamentação, é importante salientar que ela não possui riscos para o bebê, a menos que as mamas estejam com alguma lesão presente (MADRID L, et al., 2016).

A sífilis congênita é uma doença que pode ser prevenida e, diante de um diagnóstico precoce e tratamento adequado nas gestantes e seus parceiros sexuais, é possível que ocorra uma eliminação total da bactéria causadora através de estratégias efetivas de prevenção que devem ser implementadas na sociedade (PETERMAN TA e CHA S, 2018). Na maior parte dos casos ou a mãe não possuiu um pré-natal completo, não sendo testada para sífilis durante a gestação, ou ela não recebeu tratamento adequado para sífilis antes ou durante o período gestacional (PEELING RW, et al., 2017).

\section{Histopatologia do Treponema pallidum}

Para a visualização da bactéria causadora da sífilis é necessária a utilização da microscopia de campo escuro. Isso se dá pelo fato de que, devido a sua largura limitada, é impossível que ela seja visualizada por meio da microscopia direta. Pelo método de campo escuro é possível visualizar os treponemas vivos. Eles possuem forma de espiral, normalmente, aparecem como se estivessem movendo para frente e para trás. $O$ T. pallidum também pode ser visualizado usando coloração imunofluorescente e colorações especiais de prata. No exame histológico da placenta, ela também pode apresentar sinais alterações vasculares proliferativas, de vilosidades hipercelulares aumentadas e vilosite aguda ou crônica (COOPER JM e SANCHEZ PJ, 2018). 


\section{Gestação e o diagnóstico de sífilis}

Segundo o Ministério da Saúde, a assistência pré-natal inclui a recomendação para a realização de exames para o rastreio de sífilis na gestante nos primeiro e terceiro trimestres de gestação e duante o préparto. Ele pode ser feito através de testes imunológicos, como o teste não treponêmico (VDRL) e o teste treponêmico (teste rápido para sífilis). O recomendado é que investigação seja iniciada pelo teste rápido e confirmada através do VDRL, sendo os dois exames utilizados também para acompanhamento (KENYON CR, et al., 2016).

Uma vez diagnosticada, a gestante deverá ser rigorosamente tratada com Penicilina de acordo com o estágio da doença e a notificação compulsória da doença deverá ser realizada. O tratamento dessa doença pode ser iniciado antes do resultado do segundo teste, sendo definido apenas com um teste reagente, do tipo treponêmico ou não treponêmico. Quando diagnosticada corretamente e precocemente, a gestante com sífilis pode ser facilmente tratada, evitando que ocorra a contaminação da criança intraútero. (MINISTÉRIO DA SAÚDE, 2019).

\section{Manifestações clínicas da sífilis congênita}

Até $90 \%$ dos Recém-Nascidos (RNs) com sífilis congênita são assintomáticos. Diante desse cenário é necessário que os profissionais de saúde analisam a história da mãe para estimar o risco de um bebê ter sífilis congênita. As manifestações clínicas da sífilis congênita são divididas em dois grandes grupos: manifestações da sífilis congênita precoce (sinais e sintomas surgem até os 2 anos de vida) e manifestações clínicas da sífilis congênita tardia (sinais e sintomas surgem após 2 anos de vida) (RAC MWF, et al., 2020).

A sífilis congênita precoce pode afetar os diversos sistemas orgânicos do organismo da criança. As complicações podem ser gestacionais/perinatais, como aborto espontâneo, natimorto, prematuridade, baixo peso, restrição do crescimento intrauterino e hidropsia fetal; podem ser sistêmicas, se manifestando através da febre, hepatomegalia, esplenomegalia, linfadenomegalia generalizada e edema; mucocutâneas, correspondendo ao pênfigo palmoplantar, exantema maculopapular, rinite serossanguinolenta, condiloma plano e icterícia (GALVIS AE e ARRIET A, 2020).

Ainda nas manifestações precoces, a sífilis pode causar alterações hematológicas, como anemia, leucopenia/leucocitose e trombocitopenia; musculoesqueléticas, conhecidas como pseudoparalisia de Parrot, periostite, osteíte, osteocondrite, sinal de Wegner e sinal de Wimberger e também neurológicas, ocasionando anormalidades do líquor cefalorraquidiano, leptomeninge sifilítica aguda, sífilis crônica meningovascular e atraso no desenvolvimento neuropsicomotor. Dentre outras manifestações clínicas possíveis nessa fase da doença estão as lesões pulmonares (pneumonia alba) e lesões renais (síndrome nefrítica) (COOPER JM e SANCHEZ PJ, 2018).

A sífilis congênita tardia também é capaz de acometer diversos órgãos. Dentre elas estão alterações faciais, como a fronte olímpica, nariz em sela, hipodesenvolvimento maxilar e palato em ogiva; oftalmológicas, correspondendo a ceratite intersticial, coriorretinite, glaucoma secundário, cicatriz córnea e atrofia óptica; auditivas, causando perda auditiva sensorial; orofaríngeas, como os dentes de Hutchinson: incisivos medianos deformados, molares em amora e perfuração do palato duro; cutâneas, como as rágades (fissuras periorais e perinasais) e gomas (ROCHA AFB, et al., 2021).

Da mesma forma da sífilis precoce, a sífilis tardia também traz sequelas ao sistema nervoso central, gerando atraso no desenvolvimento, comprometimento intelectual, hidrocefalia, crises convulsivas, atrofia do nervo óptico e paresia juvenil; ao sistema esquelético, com tíbia em sabre, sinal de Higoumenakis (alargamento da porção esternoclavicular da clavícula), articulações de Clutton (artrite indolor), escápula escafoide (GALVIS AE e ARRIET A, 2020).

As manifestações tardias da sífilis surgem em até $40 \%$ das crianças filhos de mães não tratadas para sífilis durante a gestação. Muitas dessas sequelas podem ser prevenidas por meio do tratamento materno durante a gestação ou tratamento da criança nos primeiros três meses de vida. A maioria das manifestações clínicas da sífilis são inespecíficas e podem ser encontradas também no contexto de outras infecções congênitas. 
Portanto, diante da presença de qualquer alteração é necessário investigar possíveis diagnósticos diferenciais, a fim de estabelecer um diagnóstico preciso para um tratamento adequado (ROCHA AFB, et al., 2021).

\section{Tratamento e acompanhamento da criança acometida}

A benzilpenicilina é o tratamento de base para crianças acometidas pela sífilis congênita, podendo ser utilizada nas formas potássica ou cristalina, procaína ou benzatina. O tipo da droga que será utilizado pode variar de acordo com a titulação de teste não treponêmico da criança comparado ao da gestante, com o tratamento da mãe durante a gravidez e com os exames clínicos e laboratoriais realizados no recém-nascido. O tratamento deve ser reiniciado em casos de não negativação ou elevação do VDRL em até 18 meses (ROWE CR e JNAH AJ, 2018).

Quando a gestante não é tratada de forma adequada ou simplesmente não tratada, a sífilis congênita deve ser notificada, o rastreio de complicações deve ser realizado e o recém-nascido deve ser imediatamente tratado com Penicilina. O tratamento do RN altera de acordo com sua condição. Em um RN sem alterações, o tratamento de base é o uso de penicilina benzatina $50.000 \mathrm{UI} / \mathrm{kg}$, IM, dose única (MINISTÉRIO DA SAÚDE, 2014).

Nos casos em que há alteração liquórica, realiza-se uso de penicilina potássica/cristalina $50.000 \mathrm{UI} / \mathrm{kg}$, IV, de 12/12 horas na primeira semana de vida e de 8/8 horas após a primeira semana de vida, por 10 dias e reiniciar o tratamento se houver atraso na administração da dose superior a 24 horas. Já em qualquer alteração que não seja de líquor, é feita penicilina procaína $50.000 \mathrm{Ul} / \mathrm{kg} \mathrm{IM}$, uma vez por dia, por 10 dias e reiniciar o tratamento se houver atraso na administração da dose superior a 24 horas. Em casos de diagnósticos após um mês de idade, por sua vez, opta-se por penicilina cristalina $50.000 \mathrm{Ul} / \mathrm{kg}$, IV, de 4/4 horas por 10 dias (MINISTÉRIO DA SAÚDE, 2014).

Mesmo que a gestante tenha sido tratada adequadamente, o VDRL deve ser solicitado. De acordo com o resultado deste exame é estabelecido o manejo do recém-nascido e a conduta a ser tomada com a mãe. Nos casos em que o VDRL do RN é superior ao materno, deve-se notificar, rastrear as complicações e tratar com Penicilina. Quando o VDRL do RN está em outras diluições, o exame físico é a conduta mais apropriada (HUSSAIN AS e VAIDYA R, 2021).

Diante de um exame físico normal, independentemente do resultado do VDRL, o recém-nascido deve ser acompanhado. Já nos casos em que o exame físico se encontra alterado, a conduta pode variar de acordo com o resultado do VDRL. Nesses casos, se VDRL for reagente, deve-se notificar, rastrear complicações e tratar com Penicilina; porém, se VDRL for não reagente, outras infecções congênitas devem ser avaliadas, como no caso das TORCHS (Toxoplasmose, Rubéola, Citomegalovírus e Herpes simples) (JAAN A e RAJNIK $M, 2021)$.

Existem alguns indicativos para avaliar a falha na prevenção da sífilis congênita, sendo eles: aumento nos títulos não treponêmicos, ao longo do seguimento, em até duas diluições ou, após seis meses de idade, uma persistência da titulação reagente do teste não treponêmico. Na presença de pelo menos um desses indicativos, as crianças deverão ser notificadas para sífilis congênita e novos exames complementares deverão ser solicitados. Já em casos de tratamentos ineficazes, deve ser realizada uma reavaliação clínica e laboratorial desses pacientes que, em caso de alterações, o recomendado é que o tratamento seja reiniciado (MAGALHÃES DMS, et al., 2013).

A criança deve ser inserida no grupo de risco para desenvolvimento da sífilis congênita sintomática mesmo tendo recebido a penicilina na maternidade, sendo, portanto, fundamental um seguimento clínico rigoroso dessa criança. Dentre as ações recomendadas para esse seguimento estão: consultas ambulatoriais de puericultura, teste não treponêmico seriado na $1^{a}$ semana de vida e no $1^{\circ} \stackrel{\circ}{*} 2^{\circ}, 4^{\circ}, 6^{\circ}, 9^{\circ}, 12^{\circ}$ e $18^{\circ}$ mês e avaliações odontológica, oftalmológica e audiológica de 6 em 6 meses por um período mínimo de 2 anos. Em casos de alterações liquóricas, um novo exame deve ser realizado semestralmente até que se obtenha resultados normais (MINISTÉRIO DA SAÚDE, 2019). 


\section{Manejo da criança após exposição á sífilis}

A fim de evitar intervenções desnecessárias ao recém-nascido, o tratamento da gestante com sífilis deve ser minuciosamente estudado. Alguns itens devem ser levados em consideração para que o tratamento da gestante seja considerado adequado: o risco de reinfecção, início do tratamento até 30 dias antes do parto, a administração de penicilina benzatina com o intervalo doses de acordo com o recomendado, terapia de tratamento escolhida de acordo com o estágio clínico da doença e queda dos títulos em teste não treponêmico em pelo menos duas diluições em três meses ou de quatro diluições em seis meses após a conclusão do tratamento (KEUNING MW, et al., 2020).

Independente do histórico de tratamento da gestante, todos os recém-nascidos filhos de mãe com diagnóstico prévio de sífilis durante a gravidez devem obrigatoriamente realizar o teste não treponêmico em sangue periférico. No pós-parto imediato, a coleta do sangue periférico tanto do bebê quanto da mãe para realização do VDRL é considerado o melhor método para interpretação dos achados sorológicos do exame da criança (PEELING RW, et al., 2018).

No VDRL da criança, uma titulação maior que a titulação materna em pelo menos duas diluições é sugestivo de sífilis congênita. Mas, em contrapartida, a ausência desse achado não exclui a possibilidade do diagnóstico, portanto, é fundamental o acompanhamento de todas as crianças expostas à sífilis durante a gestação. É importante salientar que mesmo quando a mãe foi tratada incorretamente ou não tratada durante o pré-natal, as crianças receberão um diagnóstico de sífilis congênita, independentemente dos resultados dos exames complementares ou da avaliação clínica (PETERMAN TA e CHA S, 2018).

Através de uma criteriosa avaliação epidemiológica, clínica, laboratorial e imaginológica do recém-nascido, a sífilis congênita precoce pode ser identificada. Ainda na maternidade, essas crianças quanto ao resultado do do teste não treponêmico, aos exames complementares e às manifestações clínicas (MAGALHÃES DMS, et al., 2013).

As alterações causadas pelo Treponema pallidum podem ser diagnosticadas precocemente através da realização de alguns exames complementares, como hemograma completo, teste treponêmico, TGO, TGP, albumina, bilirrubina total e frações, sódio, potássio, magnésio, análise de líquor cefalorraquidiano, neuroimagem e radiografia de ossos longos e tórax. O líquor pode ser considerado alterado quando um dos seguintes critérios é preenchido: células superiores a $25 / \mathrm{mm}^{3}$, proteínas maiores que $150 \mathrm{mg} / \mathrm{dl}$ e VDRL reagente (AKHTAR F e REHMAN S, 2018).

\section{CONSIDERAÇÕES FINAIS}

A sífilis congênita é uma doença que passa da mãe contaminada pela sífilis para o feto através da placenta durante a gestação. Sua transmissão pode ocorrer em qualquer fase da gravidez, sendo mais comum no início da gestação. Em contrapartida, o risco da doença é menor nesta fase, sendo, a probabilidade de transmissão e o risco, inversamente proporcionais. As manifestações clínicas são divididas em precoces, ocorrem até os 2 anos de idade e em tardias, surgem após os 2 anos de vida da criança. A sífilis congênita pode ser prevenida através do pré-natal onde são realizados o diagnóstico e o tratamento da gestante. Uma vez que não foi possível evitar a transmissão da sífilis para o recém-nascido, este deverá ser rigorosamente tratado e acompanhado na tentativa de minimizar ao máximo a repercussão da doença na vida da criança.

\section{REFERÊNCIAS}

1. AKHTAR F, REHMAN S. Prevention of Congenital Syphilis Through Antenatal Screenings in Lusaka, Zambia: A Systematic Review. Cureus, 2018; 10(1): e2078.

2. COOPER JM, SANCHEZ PJ. Congenital syphilis. Seminars in Perinatology, 2018; 42(3): 176-184.

3. GALVIS AE, ARRIET A. Congenital Syphilis: A U.S. Perspective. Children, 2020; 7(11): 203.

4. HUSSAIN AS, VAIDYA R. Congenital Syphilis. StatPearls Publishing, 2021; 1(2): 1-4.

5. JAAN A, RAJNIK M. Torch Complex. StatPearls Publishing, 2021; 1(3): 1-5. 
6. KENYON CR, et al. The Global Epidemiology of Syphilis in the Past Century - A Systematic Review Based on Antenatal Syphilis Prevalence. PLOS Neglected Tropical Diseases, 2016; 10(5): e0004711.

7. KEUNING MW, et al. Congenital syphilis, the great imitator-case report and review. The Lancet Infectious Diseases, 2020; 20(7): e173-e179.

8. MADRID L, et al. Congenital and perinatally-acquired infections in resource-constrained settings. Expert Review of Anti-infective Therapy, 2016; 22:35.

9. MAGALHÃES DMS, et al. Sífilis materna e congênita: ainda um desafio. Cadernos de Saúde Pública, 2013, 29(6): 1109-1120.

10. MINISTÉRIO DA SAÚDE. Atenção à saúde do recém-nascido: guia para os profissionais de saúde. Ministério da Saúde, Secretaria de Atenção à Saúde, Departamento de Ações Programáticas Estratégicas, 2014. Disponível em: https://bvsms.saude.gov.br/bvs/publicacoes/atencao_saude_recem_nascido_v1.pdf. Acesso em 12 out 2021.

11. MINISTÉRIO DA SAÚDE. Protocolo Clínico e Diretrizes Terapêuticas para Prevenção da Transmissão Vertical do HIV, Sífilis e Hepatites Virais. Ministério da Saúde, Secretaria de Vigilância em Saúde, Departamento de Doenças de Condições Crônicas e Infecções Sexualmente Transmissíveis, 2019. Disponível em: file:///C:/Users/medico/Downloads/miolo_pcdt_tv_08_2019.pdf. Acesso em 12 out 2021.

12. PEELING RW, et al. Syphilis. Nature Reviews Disease Primers, 2017; 12(3): 17073.

13. PETERMAN TA, CHA S. Context appropriate interventions to prevent syphilis: a narrative review. Sexually Transmitted Diseases, 2018; 45(9SSupl1): S65-S71.

14. RAC MWF, et al. Congenital syphilis: A contemporary update on an ancient disease. Prenatal Diagnosis, 2020; 40(13): 1703-1714.

15. ROCHA AFB, et al. Complications, clinical manifestations of congenital syphilis, and aspects related to its prevention: an integrative review. Revista Brasileira de Enfermagem, 2021; 74(4): e20190318.

16. ROWE CR, JNAH AJ. Congenital Syphilis: A Discussion of Epidemiology, Diagnosis, Management, and Nurses' Role in Early Identification and Treatment. Advances in Neonatal Care, 2018; 18(6): 438-445. 